\title{
Contact-deformation mechanics of the interaction of lubricated surfaces
}

\author{
A Dykha ${ }^{1}, \mathrm{~S}$ Posonsky ${ }^{1}$ \\ ${ }^{1}$ Khmelnitskyi National University, Khmelnitsky, Ukraine \\ tribosenator@gmail.com
}

\begin{abstract}
The questions of the influence of the lubricant on the conditions of deformation in the contact of solids are considered. The steel spherical specimen was pressed into a greased and dry metal surface. The microstructures of the structure of the surface layer are studied under various conditions of deformation. The diagrams of the relative deformation of the surface layers are constructed. The mechanism of the formation and distribution of internal stresses for dry and greased contact is described. Experimental studies of the introduction of a moving steel indenter into a lubricated surface have been carried out. The relationship of the deformation mechanisms of lubricated surfaces with their wear resistance is determined
\end{abstract}

\section{Introduction}

Most of the machines' friction units operate in the conditions of boundary lubrication of contacting surfaces. While designing and improving these units, it is necessary to determine the force conditions of contact interaction and, first of all, normal pressures and shear stresses. In the condition of lubricant's presence, pressure calculation is possible only if there is data on the normal deformation properties of surfaces coated with a layer of a lubricating film. The research on the influence of the lubricating medium on the deformation during static indentation of the indenter in the material surface is well known and presented in [1]. The analysis of the received results shows that in all cases, the indenter penetration (deformation) into a lubricated surface is larger than into a dry one. the technology there is a continuous process of improving the machines and their components.

\section{Literature review}

The study of the influence of loading conditions in contact in the presence of lubricants has been considered in a number of scientific papers. By using the pendulum method, described in monograph [1], it was established that under conditions of viscous friction, oscillation attenuation curve on the elastic layer of molecules has a linear law. However, the oscillation attenuation curve on an unordered layer of lubricant is characterized by the logarithmic law of damping. These data were obtained experimentally and the study lacks a theoretical description of the mechanisms of viscous friction, which explain the results received. Article [2] examined experimentally and theoretically free fluctuations of a physical pendulum, which rests with two balls on a flat surface. The theory of damping fluctuations proposed in the study for evaluating the energy losses is of a phenomenological nature and is not based on a strict mathematical description of the mechanisms of fluctuation process, which makes it difficult to give the quantitative assessment of losses caused by friction. In paper [3], a differential equation for the swinging pendulum is obtained, taking into account the deformation of a surface and a deformation component of the friction force. However, the authors examined only a particular case of small deformations, commensurate with the dimensions of contact area of the ball 
support of a pendulum. This solution is not applicable for describing the macro-displacements in the support of a pendulum, characteristic for the actual friction units of sliding in machines. Authors of article [4] conducted a nonlinear regression analysis based on the results of experimental studies into the damped oscillations of a physical pendulum, which made it possible to formulate the criteria for choosing a theoretical model of the dissipation of viscous friction. However, the experiments were performed for the conditions of balls rolling on the support, that is, for the small areas of contact in comparison with dimensions of the balls, which also does not make it possible to adequately utilize the obtained results for evaluating the characteristics of viscous friction in cylindrical sliding supports (bearings). A calculation-experimental method for determining tangential contact frictional stresses using a variational principle was proposed in work [5]. In this case, a quadratic functional of deviation of the experimental function of tangential stresses from the equilibrium condition was constructed. The authors of [6] proposed theoretical dependences for a wear testing method using a standard fourball scheme with determination of wear resistance parameters. The approximating function of the wear spot diameter on the friction path obtained by the results of wear tests was taken as the base of the method. The general methodology of this study can be used to develop a theory of test methods for other geometric schemes. In paper [7] to identify parameters of wear resistance in the wear model, a calculation-experimental method for determining calculated dependences of wear resistance parameters was developed on the basis of the wear test by the «cone - three balls» scheme. The results of wear tests of bronze conical specimens with a variable wear spot and two values of sliding velocity were taken as a base. The experimental method for measuring the characteristics of friction was applied in article [8] for examining special features of high-speed friction under conditions of thermal loading and at limited lubrication. The criterion of damping the friction fluctuations proposed in this case is not applicable to the case of viscous friction, where such fluctuations are practically absent as a result of the damping action of a layer of lubricant. Paper [9] employed a pendulum method in order to solve a problem on determining the contact rigidity of steel spherical models at dynamic loading using the system of differential equations. However, the tangential component of contact stresses from the frictional forces in this case was not considered.

\section{Methodology and research results in conditions of the static contact}

Experimental studies of the deformations of lubricated and dry contact were carried out by means Brinell's test. The indentation was carried out on a hardness tester with a force of $105 \mathrm{~N}$ with a steel ball from ShKh15 $\boldsymbol{d}=10 \mathrm{~mm}$. Simultaneously, prints were made in conditions of dry and lubricated contact. To analyze the magnitude of the strains, the grid method was used. The strain grid lines were made photos of and enlarged by means a digital camera (Fig. 1).

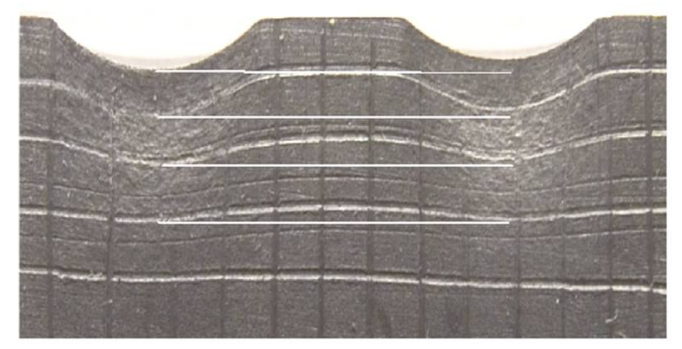

Figure 1. The grid lines deformation

The grid lines through the lower points along the axis of the deformation of the lubricated surface show a larger degree of deformation compared to deformation of a dry surface. Based on the measurements of the grid lines, we obtained diagrams of the relative deformation of the material in depth along the axis of the holes. Relative deformation diagrams for lubricated and dry contact are shown in Fig. 2. 


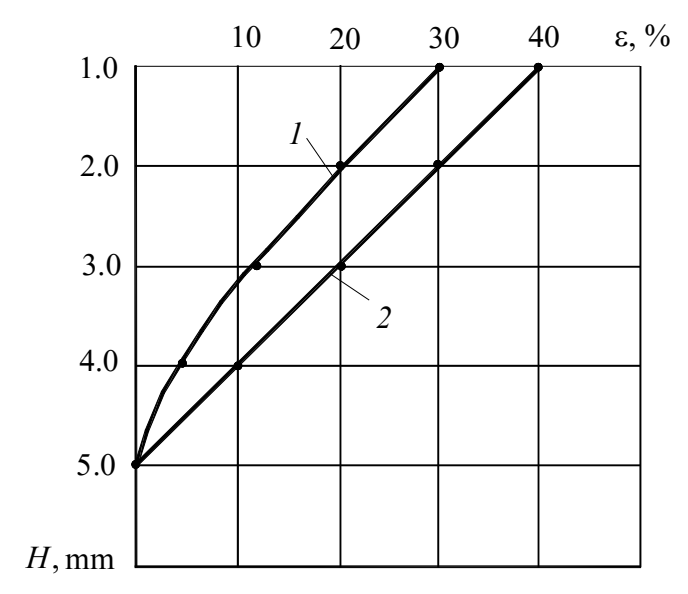

Figure 2. Relative deformation diagram: 1 - dry surface; 2 - lubricated surface

Deformation diagrams' analysis shows that the degree of relative deformation of the material in depth is larger for a lubricated contact. In the case, the dependence of deformation on depth for a lubricated contact is linear in comparison with nonlinear deformation for dry contact.

To analyze the structure of the material in the result of deformations in a lubricated and dry contact, micrometric studies were carried out. Pictures of the material structure in the zone of the deformation holes for lubricated and dry contact are shown in Fig. 3.

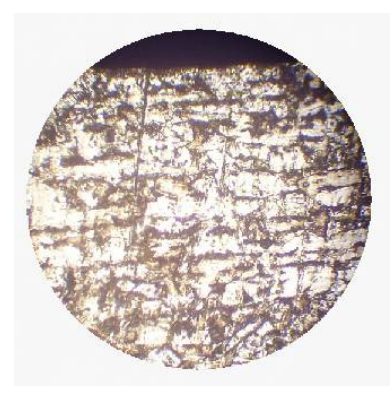

Dry contact

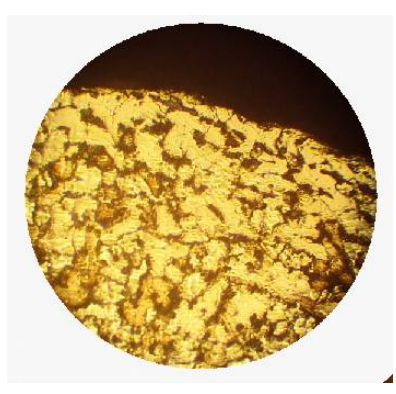

Lubricated contact

Figure 3. Material microstructure in the zone of deformation hole for dry and lubricated contact

In both cases of steel sample surface deformation, grain elongation is observed near the surface. However, the depth of the layer of deformed grains and the distribution of the deformation depth over the surface of the hole are different. The depth of the grain deformation layer for dry contact is maximum in the center of the hole and decreases to its edges. For lubricated contact, the depth of the grain deformation layer is steady over the entire surface of the deformation hole. Such a difference in the surface layer material deformation determines its stressed state in the case of dry and lubricated contact. Let us analyze the stress state of the surface layer during the contact interaction of the ball and the plane. During the contact interaction of the ball with the surface through a lubricating film, the metal is evenly deformed in the radial direction, while the surface layer is elongated. After removing the load, the elastically deformed subsurface layer tends to return the surface layer to its initial position, creating in it evenly distributed compression stresses (Fig. 4, a). 


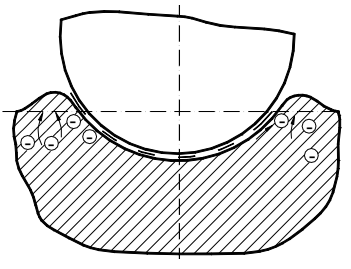

a

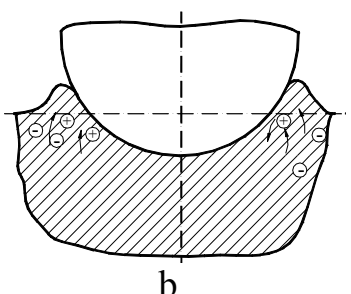

Figure 4. The scheme of internal stresses' formation: $a$ - lubricated contact; $b$ - dry contact

In case of dry contact (Fig. 4, b), the surfaces in the contact adhere due to high contact friction. Deformation of the material occurs normally deep into the surface. In this case, the subsurface layer in which compression stresses arise, is more intensively deformed, and in the surface - tensile stress.

Thus, the elastic-plastic non-lubricated contact contributes to a more intense accumulation of defects (dislocations, vacancies) in the surface layer and the concentration of internal stresses in comparison with the lubricated contact. The destruction of surface layers in the process of wear is known to begin in places of stress concentration. Thus, to reduce wear, the contact conditions of the surfaces should ensure the deconcentration of internal stresses and their uniform redistribution in the volume to reduce crystalline defects. The analysis of the deformation conditions for lubricated and dry contact during the static introduction of a spherical indenter showed the following:

1) indenter penetration depth into the lubricated surface is larger than into dry one on average by 8 $10 \%$;

2) relative deformation deep into the surface of the material along the axis of the deformation hole is described by a linear regularity and non-linear for dry contact, which indicates an even decrease in stresses deep into the lubricated surface;

3) uniform distribution of the layer of deformed grains over the deformation zone for a lubricated contact indicates the uniform distribution of stresses over the deformation zone;

4) uniform distribution of stresses in the contact of lubricated surfaces reduces the appearance of imperfections in the crystal structure (vacancies, dislocations) in the surface layer. This contributes to increased wear resistance compared to contacting surfaces without lubrication.

\section{Indenter movement with lubricated and dry contact of friction surfaces}

Further studies of dry and lubricated surfaces' deformation properties were carried out for a moving indenter. Indenter 2 (Fig. 5) was loaded with axial load through a system of levers 3. Sample 1 with a lubricant sample moved in the longitudinal direction.

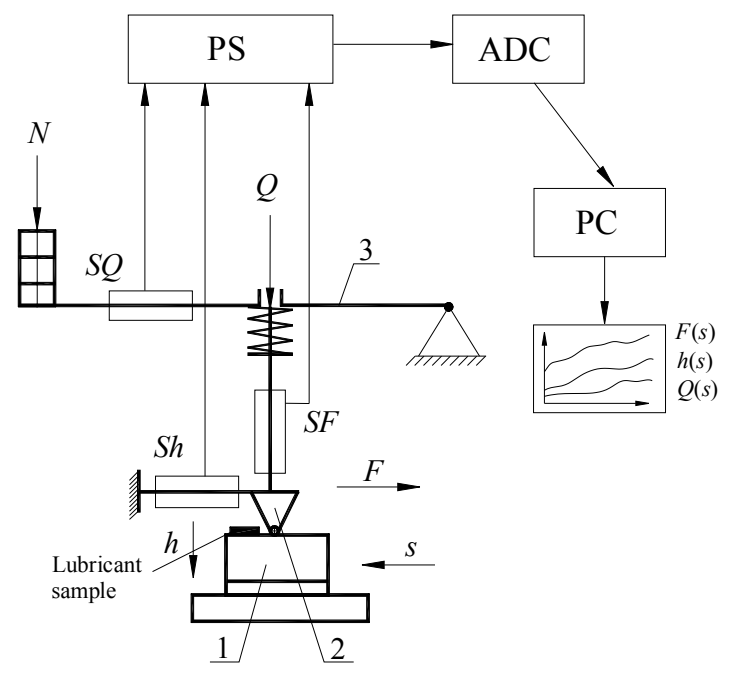

Figure 5. Test scheme 
The indenter penetrated into a dry surface and moved to the entrance to the lubricated zone. Herewith, the penetration depth and the movements resistance measurements were made.

A standard ball with a diameter of $2.5 \mathrm{~mm}$ made of ball-bearing steel was used as an indenter. The limits of the load, acting on the indenter made up $0.5 \ldots 3 \mathrm{~N}$. The accuracy of measuring the load acting on the indenter was $0.02 \mathrm{~N}$. The samples were made of brass LC 59-1 as a standard microsection. The system for measuring the resistance to displacement consisted of a strain gauge with a sensor (measurement accuracy $0.02 \mathrm{~N}$ ). The penetration depth measurement system included a strain gauge with a sensor with a measurement accuracy of $1 \mathrm{mkm}$. The signals from the strain gauges through the strain gauge were transmitted to an analog-and-digital converter and a computer. The voltage from the strain gauges in the time coordinate with a division value of $0.5 \mathrm{~s}$ was reproduced on the display screen. This corresponded to the indenter moving by a linear value of $28 \mathrm{mkm}$. The indenter was introduced into the dry surface of the sample with a constant force of $2.5 \mathrm{~N}$. The sensor readings were taken for $100 \mathrm{~s}$, which corresponded to a $5.6 \mathrm{~mm}$ displacement of the sample relative to the indenter. Fig. 6 shows diagrams of parameter changes for two lubricants types.

Shell Helix 15W-40

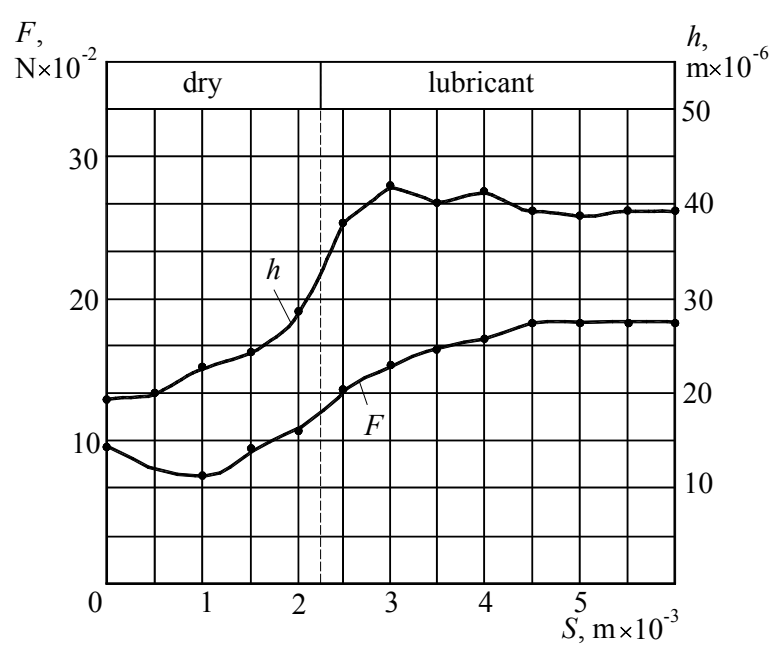

Q8 Formula 15W-40

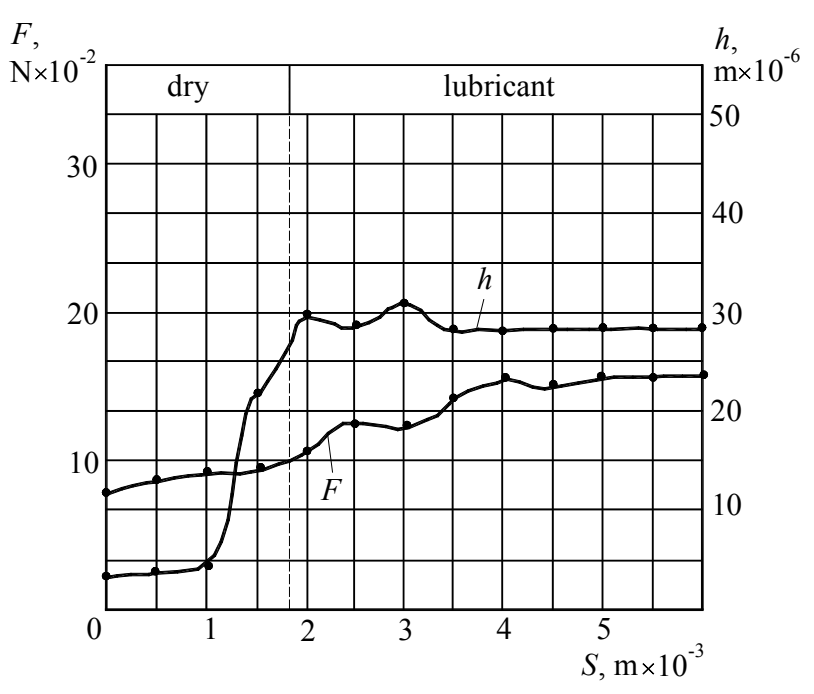

Figure 6. Diagrams of changes of penetration depth $h$ and resistance force $F$ of the indentor on the size of moving $S$ on dry and lubricated sample surface. 
Consequently, it was found out that the penetration depth of the indenter increases when passing through the lubricated area, which indicates its easier deformation compared to penetration into a dry surface. The resistance force during the passage of the indenter along the lubricated area also increases, which is explained by the indenter deepening. This leads to an increase in the volume of the deformable material and the resistance force.

\section{Conclusions}

- For studying the normal deformation properties of lubricated surfaces, a comparative analysis of lubricated and dry surface deformation conditions was carried out during tests by means of a Brinell test.

- The analysis of the deformation conditions for the lubricated contact showed that the deformation deep into the surface of the material along the axis of the deformation hole is described by a linear regularity and a non-linear one for dry contact. A uniform distribution of deformed grains for a lubricated contact indicates a uniform distribution of stresses and a decrease in the concentration of structural defects. This accordingly contributes to increased wear resistance;

- It is found out that the resistance force increases when the indenter moves along the lubricated area, which is explained by the indenter deepening. The results of observations showed an increase in the width of the grooves of the tracks in all cases after entering the blurred area by 20 - 35 percent.

- The results obtained show that more intense deformation of irregularities in a lubricated contact helps to accelerate the running-in process and reduce contact pressures of contact areas.

- Together with the traditional mechanism for reducing wear as a result of the formation of adsorbed layers on lubricated friction surfaces, the contact-deformation mechanism for reducing the wear of lubricated surfaces is justified.

\section{References}

[1] Bhushan, B. (2013). Introduction to Tribology. Second edition. New York: John Wiley \& Sons, $721 \mathrm{p}$.

[2] Dzhilavdari, I., Riznookaya, N. (2008). An experimental assessment of the components of rolling friction of balls at small cyclic displacements. Journal of Friction and Wear, 29(5), 330-338.

[3] Dzhilavdari, I., Riznookaya, N. (2007). Specific features of the dynamics of small-amplitude pendulum swings in the presence of hysteresis friction. Friction and Wear, 28(2), 143-150.

[4] Dzhilavdari, I., Riznookaya, N. (2008). Studies of the dynamics of free micro oscillations of a pendulum supported by two balls. Journal of Friction and Wear, 29(1), 1-8.

[5] Dykha, A.V., Kuzmenko, A.G. (2016). Distribution of friction tangential stresses in the Courtney-Pratt experiment under Bowden's theory. Journal of Friction and Wear, 37 (4), 315-319. doi: $10.3103 / \mathrm{s} 1068366616040061$

[6] Dykha, A.V., Kuzmenko, A.G. Solution to the problem of contact wear for four-ball weartesting scheme. Journal of Friction and Wear, 2015, 36(2), 138-143. doi: 10.3103/S1068366615020051

[7] Dykha, A., Sorokatyi, R., Makovkin, O., Babak, O. (2017). Calculation-experimental modeling of wear of cylindrical sliding bearings. Eastern-European Journal of Enterprise Technologies, 5 (1 (89), 51-59. doi: 10.15587/1729-4061.2017.109638.

[8] Sorokatyi, R. , Dykha A. (2015). Analysis of processes of tribodamages under the conditions of high-speed friction. Journal of Friction and Wear, 36 (5), 422-428.

[9] Sherif, H.A., Almufadi, F.A. (2016). Identification of contact parameters from elastic-plastic impact of hard sphere and elastic half space. Wear, 368-369 (3), 358-367. 